\title{
Loss Strategies for Competing AIMD Flows *
}

\author{
Eitan Altman \\ INRIA, B.P. 93, 2004 Route des Lucioles, 06902 Sophia-Antipolis Cedex, France \\ Rachid El Azouzi \\ Université d'Avignon et des Pays de Vaucluse (IUP), LIA - CERI, 339 chemin \\ des Meinajariès, BP 1228, 84911 Avignon Cedex 9, France \\ David Ros \\ GET/ENST Bretagne, rue de la Châtaigneraie, CS 17607, 35576 Cesson Sévigné \\ Cedex, France \\ Bruno Tuffin \\ IRISA/INRIA Rennes, Campus de Beaulieu, 35042 Rennes Cedex, France
}

\begin{abstract}
We study in this paper two competing AIMD flows that share a common bottleneck link. When congestion occurs, one (or both) flows will suffer a loss that will cause its throughput to decrease by a multiplicative factor. The identity of the flow that will suffer a loss is determined by a randomized "loss strategy" that may depend on the throughputs of the flows at the congestion instant. We analyze several loss strategies: the one in which the identity of the flow experiencing the loss is independent of the current throughput and the one in which the flow with the largest throughput is to suffer the loss; this is compared with the strategy that assigns loss probabilities proportionally to the throughputs (thus a flow with a larger throughput has a larger loss probability). After deriving some results for the general asymmetric case, we focus in particular on the symmetric case and study the influence of the strategy on the average throughput and average utilization of the link. As the intuition says, a strategy that assigns a loss to a flow with a higher throughput is expected to give worse performance since the total instantaneous throughput after a loss is expected to be lower with such a strategy. Surprisingly, we show that this is not the case. We show that the average throughput and average link utilizations are invariant: they are the same under any possible strategy; the link utilization is $6 / 7$ of the link capacity. We show, in contrast, that the second moment of the throughput does depend on the strategy.
\end{abstract}


Key words: Additive increase multiplicative decrease (AIMD) algorithm, TCP, fairness, pricing.

\section{Introduction}

The mathematical analysis of the performance of TCP has been a major research area in networking. Different types of approaches have been suggested and validated. On the one hand, there have been models focusing on a single connection that is subject to some exogenous loss process (which does not depend on that connection), see e.g. [2]. This approach is appealing when there is a large amount of traffic, so that we can neglect the effect of the single connection on events that cause losses. An alternative approach is necessary when the window increase of a connection is itself a central cause for losses. This occurs typically when a small number of connections compete over bandwidth, say, at a bottleneck link. A main mathematical approach for studying this situation has been to study several connections sharing a bottleneck, and then make the simplifying assumption that all connections reduce their windows simultaneously upon congestion [3-5]. With this approach, it has been shown [4] that the throughput achieved by a TCP connection is inversely proportional to $R T T^{\alpha}$ with $1<\alpha<2$, where $R T T$ is the two-way propagation delay of the connection. However, it turns out that in practice this assumption does not hold, except for drop tail buffers and connections with similar Round Trip Times (RTTs) [6]. Indeed, traces in [5] (e.g. Fig. 5) show that the synchronization assumption is invalid for asymmetric connections for a drop tail buffer.

Instead of considering synchronization, two modeling approaches have been developed for determining which connection will suffer a packet loss. In the model of Baccelli and Hong [7], the probability that a connection will lose a packet is a constant: it does not depend on its current throughput. As argued in [8], such an assumption is valid in describing AIMD protocols in which packet transmission rates are constant, and the throughput is varied by changing the packet size. The use of fixed loss probabilities, with different probabilities for different flows, can be justified by a pricing-based service differentiation policy where smaller loss probabilities would be associated with sessions with a

\footnotetext{
‡ A shorter version of this paper appeared in the Proceedings of the Third IFIPTC6 Networking Conference [1].

Email addresses: Eitan.Altman@sophia.inria.fr (Eitan Altman), Rachid.Elazouzi@lia.univ-avignon.fr (Rachid El Azouzi), David.Ros@enst-bretagne.fr (David Ros), Bruno.Tuffin@irisa.fr (Bruno Tuffin).
} 
higher willingness to pay. An alternative model has been considered in [9] in which the probability that a connection loses a packet is proportional to the throughput at the congestion instant. This is called the "proportional strategy". As validated by simulations [10], this model is appropriate for standard TCP where packet size is constant.

Motivated by these two approaches, we raise the question of what is the throughput of an AIMD flow as a function of the strategy that determines which flow loses a packet at a congestion instant. We focus on the simple scenario of two competing flows.

Our findings are as follows. We first study the constant probability model from [7]. In that paper, a linear set of stochastic recursive equations has been introduced for obtaining the throughput, in which the state variables correspond to the flows' throughputs after a loss. In this paper we present an alternative set of stochastic recursive equations in which the states correspond to the throughput just before the loss occurs. We show that our approach allows us to reduce the dimensionality of the system by one, so in particular, the case of two flows can be described by a one-dimensional state equation. This allows us to obtain an explicit expression for the throughput in the general asymmetric case for the constant probability model. As a corollary of this result, it is seen in the symmetric case that the link utilization is $6 / 7$ of its capacity.

We then study a new strategy in which the flow with the largest instantaneous throughput is the one to lose a packet at congestion instants. Surprisingly, we obtain the same average throughput and link utilization in the symmetric case as for the constant probability model. Moreover, this is the same utilization also obtained for the proportional strategy. This motivated us to examine the behavior of an arbitrary strategy. Our main finding is that although the expectation of throughputs at loss instants depend on the strategy, the average throughput is an invariant quantity for the case of symmetric flows.

We finally derive a general expression for the second moment of the throughput and compare the performance of the three strategies mentioned above in the symmetric case, in order to find out which one has the smallest throughput variability.

The structure of the paper is as follows. In Section 2 we study the throughput of the constant loss strategy, whereas the Largest Throughput Loss (LTL) strategy is analyzed in Section 3. Section 4 then presents some numerical experimentations and comparisons between the strategies. Section 5 studies the average throughputs in the symmetric setting under an arbitrary strategy and obtains the invariance property. Section 6 then provides an expression for the second moment of the throughput under an arbitrary strategy and a comparison for the three aforementioned strategies. We end with a concluding 
section.

\section{Fixed loss probabilities: model and analysis}

\subsection{Basic definitions and assumptions}

This model is based on [7] where an additive increase, multiplicative decrease (AIMD) model is used to describe the joint throughput evolution of a set of TCP sessions sharing a common router bottleneck.

In full generality, let $N$ be the number of AIMD sessions competing for bandwidth, and $C$ the capacity of the bottleneck router. Let $T_{n}$ be the $n$-th congestion epoch and $\tau_{n+1}=T_{n+1}-T_{n}$. Let also $\eta_{i}$ be the additive increase rate for session $i$ and $\beta_{i}$ be its multiplicative decrease rate. Usually, $\beta_{i}=1 / 2 \forall i$ and $\eta_{i}$ is taken as the square inverse of the round trip time of session $i$. We consider here $Y_{n}^{(i)}$, the throughput of session $i$ before the $n$-th congestion epoch, instead of $X_{n}^{(i)}$, the throughput after the $n$-th congestion epoch like in [7].

Denote by $\bar{Y}^{(i)}$ session $i$ 's mean throughput. As in [7], let $a_{n}^{(i)}$ be a Bernoulli random variable with value 1 if session $i$ experiences a loss at the $n$-th congestion epoch, and 0 otherwise, so that $\mathbb{E}\left[a_{n}^{(i)}\right]=p_{i}$. Note that the $a_{n}^{(i)}(1 \leq i \leq N)$ are correlated to make sure that at least one packet is lost at each congestion time.

We have

$$
Y_{n+1}^{(i)}=\gamma_{n}^{(i)} Y_{n}^{(i)}+\tau_{n+1} \eta_{i}
$$

where $\gamma_{n}^{(i)}=\left(1-a_{n}^{(i)}\right)+\beta_{i} a_{n}^{(i)}$.

As in [7], we assume here that there is a loss as soon as the router capacity is reached, i.e., as soon as

$$
\sum_{i=1}^{N} \gamma_{n}^{(i)} Y_{n}^{(i)}+\tau_{n+1} \sum_{i=1}^{N} \eta_{i}=C
$$

This assumption will allow us to derive the throughput at the different congestion epochs.

\subsection{Practical justification: pricing for service differentiation}

An important question is the justification and choice of the different loss probabilities for different flows. Fixing different probabilities would actually allow 
service differentiation, which becomes compulsory in a congested network with various QoS requirements. To make sure that users do not always choose the best QoS, a pricing scheme has to be associated with the loss probability choice. For surveys on pricing schemes in telecommunication networks, the reader can see e.g. [11-13].

For instance we can assume that each session tries to optimize its utility

$$
U_{i}(u)=\bar{Y}^{(i)}-q u
$$

where

- $u$ is session $i$ 's willingness to pay (that will actually be charged), with optimal value $u_{i}$, so that session $i$ loss probability is given by

$$
p_{i}=f^{(i)}\left(u_{1}, \cdots, u_{N}\right)
$$

such that $\sum_{i=1}^{N} f^{(i)}\left(u_{1}, \cdots, u_{N}\right) \geq 1$. We just have to make sure that at least one loss is experienced (meaning that the random variables describing whether a session experiences a loss are correlated). For instance, we can choose

$$
p_{i}=\frac{u_{i}^{-1}}{\sum_{j=1}^{N} u_{j}^{-1}},
$$

meaning that the loss probability for session $i$ is inversely proportional to its willingness to pay and that exactly one session will suffer from a loss at each congestion epoch.

- $q$ is a constant representing the relative weight between the charge and the throughput (that is fixed by the network manager, in order to maximize the network revenue),

\subsection{Computation of the average throughput}

The goal of this subsection is to derive the average throughput of a session in terms of the loss probabilities when the number of sessions is $N=2$.

First, using (2), we get the time between the $n$-th and $(n+1)$-th congestion epochs

$$
\tau_{n+1}=\frac{C-\sum_{i=1}^{N} \gamma_{n}^{(i)} Y_{n}^{(i)}}{\sum_{i=1}^{N} \eta_{i}}
$$

Using this relation we are able to derive a closed-form of the average throughput $\bar{Y}^{(1)}$ of session 1 . The average throughput $\bar{Y}^{(2)}$ of session 2 can be obtained in the same way (or by switching the indexes 1 and 2 in the following formula). 
Proposition 1 Assume that $N=2$. If we denote $p_{12}=\mathbb{E}\left(a^{(1)} a^{(2)}\right), \xi_{i}=$ $\eta_{i} /\left(\eta_{1}+\eta_{2}\right), \alpha_{i}=1-\beta_{i}, p_{i}^{\prime}=\alpha_{i} p_{i} \forall i=1,2$ and $p_{12}^{\prime}=\alpha_{1} \alpha_{2} p_{12}$, we have that

$$
\begin{gathered}
\bar{Y}^{(1)}=\frac{C \xi_{1}\left(\xi_{1} p_{2}^{\prime}+\xi_{2} p_{1}^{\prime}\right)}{2 p_{1}^{\prime}}\left(\frac{\left(\xi_{1} \alpha_{2}-2\right) p_{2}^{\prime}+\left(2-\left(1+\xi_{2}\right) \alpha_{1}\right) p_{1}^{\prime}+2 \xi_{2} p_{12}^{\prime}}{\left(2 \xi_{2}-\xi_{1}^{2} \alpha_{1}\right) p_{1}^{\prime}+\left(2 \xi_{1}-\xi_{2}^{2} \alpha_{2}\right) p_{2}^{\prime}-2 \xi_{1} \xi_{2} p_{1} p_{12}^{\prime}} \times\right. \\
\left.\quad \times\left(\xi_{1} \alpha_{2}+2 \xi_{1} \frac{\left(1-\xi_{1} \alpha_{2}\right) p_{2}^{\prime}-\xi_{2} p_{12}^{\prime}}{\xi_{1} p_{2}^{\prime}+\xi_{2} p_{1}^{\prime}}\right)+2 \frac{\left(1-\xi_{1} \alpha_{2}\right) p_{2}^{\prime}-\xi_{2} p_{12}^{\prime}}{\xi_{1} p_{2}^{\prime}+\xi_{2} p_{1}^{\prime}}+\alpha_{2}\right) .
\end{gathered}
$$

The proof of this formula is provided in Appendix A.

Corollary 1 Still assuming $N=2$, the symmetric case yields

$$
\bar{Y}^{(1)}=\frac{C}{4} \frac{\left(2 p_{1}-\left(1-\beta_{1}\right) p_{12}\right)\left(2\left(1+\beta_{1}\right) p_{1}+\left(1-\beta_{1}\right)\left(1-p_{1}\right) p_{12}\right)}{p_{1}^{2}\left(3+\beta_{1}-\left(1-\beta_{1}\right) p_{12}\right)} .
$$

Proof: just replace $\eta_{2}$ by $\eta_{1}, \beta_{2}$ by $\beta_{1}$ and $p_{2}$ by $p_{1}$ in Proposition 1 .

\subsection{Sampling the loss probabilities}

The previous expressions of the average throughput are general in the sense that no special sampling structure has been used for the losses. In this section, we aim at studying how the losses can be sampled and how it impacts on the average throughput formula.

\subsubsection{Independent sampling}

As in [7], we can assume that the $a_{n}^{(i)}$ are at first generated independently, such that $\mathbb{P}\left[a_{n}^{(i)}=1\right]=\pi_{i}$, with $\pi_{i}$ given, but that the samples are restricted to the domain where at least one loss is experienced. This requires a derivation of $\pi_{i}$ in terms of the $p_{j}$.

Assuming $N=2$, we have as in [7]

$$
\left\{\begin{array}{l}
p_{1}=\frac{\pi_{1}}{1-\left(1-\pi_{1}\right)\left(1-\pi_{2}\right)} \\
p_{2}=\frac{\pi_{2}}{1-\left(1-\pi_{1}\right)\left(1-\pi_{2}\right)}
\end{array}\right.
$$


where $\pi_{i}$ is for the loss probability for user $i$, sampled independently, but reduced to the domain such that a loss is actually experienced. This gives

$$
\left\{\begin{array}{l}
\pi_{1}=p_{1}\left(\pi_{1}+\pi_{2}-\pi_{1} \pi_{2}\right) \\
\pi_{2}=p_{2}\left(\pi_{1}+\pi_{2}-\pi_{1} \pi_{2}\right)
\end{array}\right.
$$

We obtain the relation

$$
\pi_{1}=\frac{p_{1}}{p_{2}} \pi_{2}
$$

which gives (assuming $\pi_{2}>0$ )

$$
\pi_{2}=\frac{p_{1}+p_{2}-1}{p_{1}}
$$

and then

$$
\pi_{1}=\frac{p_{1}+p_{2}-1}{p_{2}}
$$

Then an assumption $p_{1}+p_{2}=1$ can not be used. Also, it seems difficult to make sure that $\pi_{1} \leq 1$ and $\pi_{2} \leq 1$ for every pair $\left(p_{1}, p_{2}\right)$. Thus this sampling procedure does not work in full generality.

\subsubsection{A single loss at congestion epochs}

The simplest way to sample is by using the relation

$$
a_{n}^{(2)}=1-a_{n}^{(1)}
$$

with $a_{n}^{(1)}$ a Bernoulli random variable such that $\mathbb{P}\left[a_{n}^{(1)}=1\right]=p_{1}$. This means that at each congestion epoch, one and only one session will see a decrease of its throughput. One way to achieve it would be for instance to consider $\forall i \in\{1,2\}, p_{i}=\frac{u_{i}^{-1}}{u_{1}^{-1}+u_{2}^{-1}}($ see Section 2.2).

We then have

$$
\begin{aligned}
p_{2} & =1-p_{1}, \\
p_{12} & =0 .
\end{aligned}
$$

Substituting these values in (4), we get 


$$
\begin{gathered}
\bar{Y}^{(1)}=\frac{C \xi_{1}\left(\xi_{1} \alpha_{2}\left(1-p_{1}\right)+\xi_{2} p_{1}^{\prime}\right)}{2 p_{1}^{\prime}}\left(\left(\xi_{1} \alpha_{2}+2 \xi_{1} \frac{\left(1-\xi_{1} \alpha_{2}\right) \alpha_{2}\left(1-p_{1}\right)}{\xi_{1} \alpha_{2}\left(1-p_{1}\right)+\xi_{2} p_{1}^{\prime}}\right) \times\right. \\
\left.\times \frac{\left(\xi_{1} \alpha_{2}-2\right) \alpha_{2}\left(1-p_{1}\right)+\left(2-\left(1+\xi_{2}\right) \alpha_{1}\right) p_{1}^{\prime}}{\left(2 \xi_{2}-\xi_{1}{ }^{2} \alpha_{1}\right) p_{1}^{\prime}+\left(2 \xi_{1}-\xi_{2}^{2} \alpha_{2}\right) \alpha_{2}\left(1-p_{1}\right)}+2 \frac{\left(1-\xi_{1} \alpha_{2}\right) \alpha_{2}\left(1-p_{1}\right)}{\xi_{1} \alpha_{2}\left(1-p_{1}\right)+\xi_{2} p_{1}^{\prime}}+\alpha_{2}\right)
\end{gathered}
$$

The symmetric case (with $p_{1}=p_{2}=1 / 2, \eta_{2}=\eta_{1}$ and $\beta_{2}=\beta_{1}$ ) yields

$$
\bar{Y}^{(1)}=\frac{\left(1+\beta_{1}\right) C}{3+\beta_{1}} .
$$

If $\beta_{1}=1 / 2$, we obtain $\bar{Y}^{(1)}=\frac{3}{7} C$, like in [9] for the proportional loss strategy.

\section{The largest throughput loss (LTL) strategy}

Let us look at the case where the session that is penalized is systematically the one with the largest throughput. We call this the "Largest Throughput Loss" (LTL) strategy. Consider the $n$-th congestion epoch, with throughputs $Y_{n}^{(1)}$ and $Y_{n}^{(2)}$ such that $Y_{n}^{(1)}+Y_{n}^{(2)}=C$. Without loss of generality, assume $Y_{n}^{(1)}>Y_{n}^{(2)}$ and that the additive increase is 1 .

\subsection{The symmetric case: the periodic solution}

We identify a periodic solution for the evolution of the system. In this regime, we assume (without loss of generality) that at time $n$, flow 1 has a larger throughput than flow 2. We seek for a regime in which at time $n+1$ the situation is reversed, and so on. This gives the following dynamics:

$$
\left\{\begin{aligned}
Y_{n}^{(1)} / 2+\tau_{n+1} & =Y_{n}^{(2)} \\
Y_{n}^{(2)}+\tau_{n+1} & =Y_{n}^{(1)} \\
Y_{n}^{(1)}+Y_{n}^{(2)} & =C
\end{aligned}\right.
$$

leading to

$$
\tau_{n+1}=\frac{1}{7} C, Y_{n}^{(1)}=\frac{4}{7} C \text { and } Y_{n}^{(2)}=\frac{3}{7} C
$$


As in the proof of Proposition 1, but due to the periodicity of the system, the average throughput is given by $S / \mathbb{E}[2 \tau]$ where $S$ is the cumulative throughput of a session between congestion epochs $n$ and $n+2$ (in one period, the throughput is going from $2 C / 7$ to $3 C / 7$ and in the other one from $3 C / 7$ to $4 C / 7)$. This gives $S=\frac{12}{98} C^{2}$, leading again to $\bar{Y}^{(1)}=\bar{Y}^{(2)}=\frac{3}{7} C$ and an average utilization of $\frac{6}{7}$ as we obtained in the previous section and as is the case in the model in [9]. Obviously, $\mathbb{E}\left[Y_{n}^{(i)}\right]$ are also the same in all three cases (and equal to $C / 2$ ). One could wonder whether in fact the distribution of the rates is independent of the way one chooses the flow to decrease the rate at $T_{n}$. Note however, that $\mathbb{E}\left[\left(Y_{n}^{(i)}\right)^{2}\right]=25 C^{2} / 98$ in our example, which is different than the value of $7 C^{2} / 26$ obtained in the regime considered in [9].

\subsection{The dynamic equations for the asymmetric case}

For each flow $i=1,2$ we have

$$
Y_{n+1}^{(i)}= \begin{cases}Y_{n}^{(i)} / 2+\tau_{n+1} \eta_{i} & \text { if } Y_{n}^{(i)}>C / 2 \\ Y_{n}^{(i)}+\tau_{n+1} \eta_{i} & \text { if } Y_{n}^{(i)}<C / 2 .\end{cases}
$$

For the case that $Y_{n}^{(i)}=C / 2$ any tie breaking rule can be considered. Combining this with the relation $Y_{n}^{(2)}=C-Y_{n}^{(1)}$ as well as $Y_{n+1}^{(2)}=C-Y_{n+1}^{(1)}$ gives

$$
\tau_{n+1}= \begin{cases}\frac{Y_{n}^{(i)}}{2\left(\eta_{1}+\eta_{2}\right)} & \text { if } Y_{n}^{(i)}>C / 2 \\ \frac{C-Y_{n}^{(i)}}{2\left(\eta_{1}+\eta_{2}\right)} & \text { if } Y_{n}^{(i)}<C / 2 .\end{cases}
$$

Substituting in (7) gives

$$
Y_{n+1}^{(i)}= \begin{cases}\frac{1}{2}\left(1+\frac{\eta_{i}}{\eta_{1}+\eta_{2}}\right) Y_{n}^{(i)} & \text { if } Y_{n}^{(i)}>C / 2 \\ \left(1-\frac{\eta_{i}}{2\left(\eta_{1}+\eta_{2}\right)}\right) Y_{n}^{(i)}+\frac{C \eta_{i}}{2\left(\eta_{1}+\eta_{2}\right)} & \text { if } Y_{n}^{(i)}<C / 2 .\end{cases}
$$

These equations can be used to obtain the exact transient behavior of the system. The average throughput can then be computed by

$$
\bar{Y}^{(i)}=\lim _{n \rightarrow \infty} \frac{\sum_{k=1}^{n} \tau_{k+1}\left(Y_{k+1}^{(i)}+\gamma_{k}^{(i)} Y_{k}^{(i)}\right) / 2}{\sum_{k=1}^{n} \tau_{k+1}}
$$

\subsection{The case $\eta_{2} / \eta_{1} \rightarrow 0$}

We consider here the case of $x \rightarrow 0$ where $x:=\eta_{2} / \eta_{1}$ and assume for simplicity that $\beta_{i}=1 / 2$. We present a heuristic argument to compute the bandwidth 
sharing.

Flow 2 will increase its rate until it reaches $C / 2$, so its trajectory at steady state will be periodic (with a period of duration of $C /\left(4 \eta_{2}\right)$ ), linearly increasing between $C / 4$ to $C / 2$. Its average throughput is $3 \mathrm{C} / 8$.

Flow 1 Fix $\Delta=\sqrt{x} / \eta_{2}$. We can view the problem as one with two time scales: flow 1 is much faster than flow 2 , so during the interval $[n \Delta,(n+$ 1) $\Delta$ ), the throughput of flow 2 can be approximated by a constant which we denote by $Y^{(2)}(n)$; assume that this constant is smaller than $C / 2$. During that interval, the throughput of flow 1 will oscillate very quickly (between half of the remaining and all the remaining bandwidth) so that it will use in average over that interval $3 / 4$ of the remaining bandwidth. Thus its average bandwidth during the interval is $(3 / 4)\left(C-Y^{(2)}(n)\right)$, and during the whole period of $C /\left(4 \eta_{2}\right)$ it will be $(3 / 4)(C-3 C / 8)=15 C / 32$.

Thus as $x \rightarrow 0$ we see that the fast flow will get $5 / 4$ of the throughput of the slow flow under the LTL strategy.

3.4 Symmetric case with more than two flows $(N>2)$ : the periodic solution

Assume now that we have $N>2$ symmetric flows. Assume that $Y_{n}^{(1)}>Y_{n}^{(2)}>$ $\cdots>Y_{n}^{(N)}$ at time $n$. This gives the following system of $N+1$ equations:

$$
\left\{\begin{array}{ccc}
Y_{n}^{(1)} / 2+\tau_{n+1} & =Y_{n}^{(N)} \\
Y_{n}^{(2)}+\tau_{n+1} & =Y_{n}^{(1)} \\
: & : & : \\
Y_{n}^{(N-1)}+\tau_{n+1} & =Y_{n}^{(N-2)} \\
Y_{n}^{(N)}+\tau_{n+1} & =Y_{n}^{(N-1)} \\
Y_{n}^{(1)}+Y_{n}^{(2)}+. .+Y_{n}^{(N)} & =\quad C .
\end{array}\right.
$$

By adding up the $N$ first equations we get

$$
N \tau_{n+1}+\sum_{i=2}^{N} Y_{n}^{(i)}+Y_{n}^{(1)} / 2=C .
$$

Since $\sum_{i=2}^{N} Y_{n}^{(i)}=C-Y_{n}^{(1)}$, the last equation becomes

$$
N \tau_{n+1}+C-Y_{n}^{(1)}+Y_{n}^{(1)} / 2=C,
$$


leading to

$$
\tau_{n+1}=\frac{Y_{n}^{(1)}}{2 N}
$$

We then obtain

$$
Y^{(i)}=Y^{(1)}\left(1-\frac{i-1}{2 N}\right) .
$$

From $\sum_{i=1}^{N} Y^{(i)}=C$, we have

$$
\begin{aligned}
Y^{(1)}\left(\sum_{i=1}^{N}\left(1-\frac{i-1}{2 N}\right)\right) & =C \\
Y^{(1)}\left(N-\frac{N-1}{4}\right) & =C \\
Y^{(1)}\left(\frac{3 N+1}{4}\right) & =C,
\end{aligned}
$$

leading to

$$
Y^{(1)}=\frac{4}{3 N+1} C
$$

From (8) we then have

$$
Y_{n}^{(i)}=\frac{2(2 N+1-i)}{N(3 N+1)} C, i=2, . . N \quad \text { and } \tau_{n+1}=\frac{2}{N(3 N+1)} C .
$$

As in the proof of Proposition 1, but due to the periodicity of the system, the average throughput is given by $S / \mathbb{E}[N \tau]$ where $S$ is the cumulative throughput of a session between congestion epochs $n$ and $n+N$. We have

$$
\begin{aligned}
S & =\frac{1}{2}\left(\left(Y_{n+1}^{(1)}-Y_{n}^{(1)} / 2\right) \tau_{n+1}+\sum_{i=2}^{N}\left(Y_{n+i}^{(1)}+Y_{n+i-1}^{(1)}\right) \tau_{n+i}\right) \\
& =\frac{\tau}{2}\left(2 \sum_{i=1}^{N-1} Y_{n+i}^{(1)}+\frac{1}{2} Y_{n}^{(1)}+Y_{n+N}^{(1)}\right)=\frac{\tau}{2}\left(2 \sum_{i=1}^{N} Y_{n+i}^{(1)}-\frac{1}{2} Y_{n}^{(1)}\right) \\
& =\frac{1}{N(3 N+1)} C\left(2 C-\frac{2}{3 N+1} C\right)=\frac{6}{(3 N+1)^{2}} C^{2} .
\end{aligned}
$$

Thus, the average throughput is given by

$$
\bar{Y}^{(i)}=\frac{3 N}{N(3 N+1)} C, \quad i=1,2, \cdots, N
$$




\section{Numerical results for the fairness in bandwidth sharing}

We study in this section the fairness in throughput as a function of the round trip times (when $N=2$ ). We recall that the square root formula of TCP as well as its refinements (see [14],[2]) predict that the throughput of a connection should be inversely proportional to its RTT. We shall compare this with the fairness obtained under our model of interacting flows.

\subsection{Constant loss strategy}

We now look at the ratio $\bar{Y}_{1} / \bar{Y}_{2}$ of average throughputs. To simplify the expressions, let us assume that $\beta_{1}=\beta_{2}=1 / 2$ and that $p_{1}=p_{2}=p \geq 1 / 2$. We also assume that the linear growth rates are inversely proportional to the square of the round trip times, i.e., $\eta_{i}=1 / R_{i}^{2}$ for $i=1$, 2. (Indeed, the window increases by one each RTT, and since the throughput is given by the window size divided by the RTT, the increase rate of the throughput is $1 / R T T^{2}$.)

We then obtain from (5) that

$$
\frac{\bar{Y}_{1}}{\bar{Y}_{2}}=\left(\frac{R_{2}}{R_{1}}\right)^{2} \frac{3 p \frac{R_{2}}{R_{1}}+5 p-2 p_{12}}{5 p\left(\frac{R_{2}}{R_{1}}\right)^{2}+3 p-2 p_{12}\left(\frac{R_{2}}{R_{1}}\right)^{2}} .
$$

If we further assume that exactly one flow will experience a loss, then we have $p_{12}=0$ and $p=1 / 2$ giving

$$
\frac{\bar{Y}_{1}}{\bar{Y}_{2}}=\left(\frac{R_{2}}{R_{1}}\right)^{2} \frac{3\left(\frac{R_{2}}{R_{1}}\right)^{2}+5}{5\left(\frac{R_{2}}{R_{1}}\right)^{2}+3} .
$$

We depict the fairness in throughputs for the fixed loss strategy in Figure 1.

As can be seen on Figure 1, the ratio of average throughputs is very close to be linear in the square of the ratio of round trip times.

\subsection{The LTL strategy}

In Figure 2 we depict the throughput ratios as a function of the ratio of the inverse of the square of RTTs for the LTL strategy. The values are obtained by computing the throughput as in Subsection 3.2. We observe that although in general the throughput has a tendency to increase as the corresponding RTT 




Fig. 1. The ratio $\bar{Y}_{1} / \bar{Y}_{2}$ as a function of the ratio $R_{2} / R_{1}$ for the constant loss strategy.

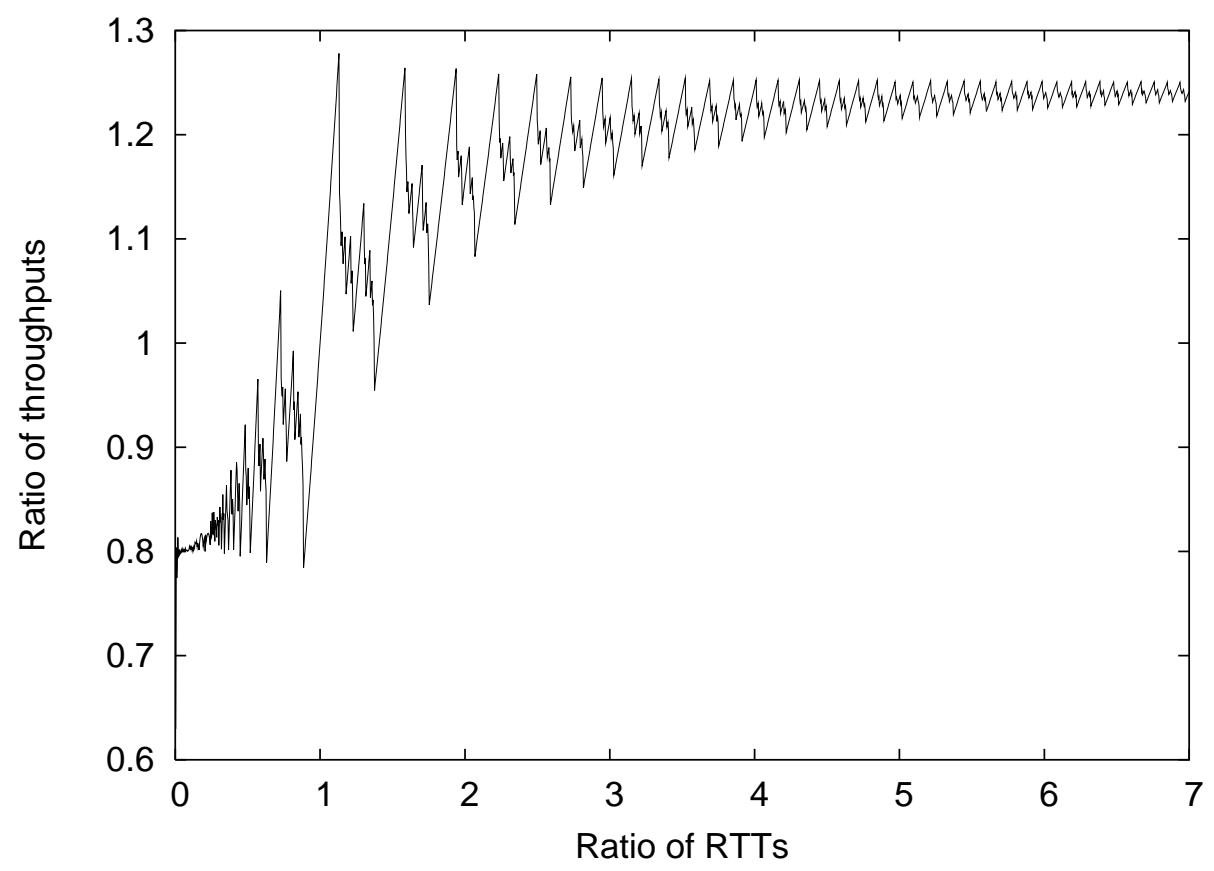

Fig. 2. The ratio in throughput as a function of the ratio of $R T T^{-1}$ for the LTL strategy.

decreases, we see that the throughput curve is quite irregular and fractal, and locally there are many points where the opposite behavior is observed: increasing the RTT of a flow results in increasing its throughput. This can perhaps be explained in part by changes in the periodicity of the steady state behav- 
ior and in other discrete nature behavior. The analysis of this phenomenon is beyond the scope of this paper. We note that other fractal aspects of AIMD flows in networks with several nodes have already been reported in [15]. We finally observe that as the RTT of a flow becomes negligible with respect to the other, its share of the throughput converges to $5 / 4$ of the throughput of the other flow, as predicted in Section 3.3.

\subsection{Comparisons}

We first observe that the throughput sharing in the LTL strategy is much more fair than in the probabilistic sharing: it is much less sensible to the differences in RTT. Indeed, a flow with 3 times smaller RTT gets only 1.21 times more throughput in the LTL strategy, whereas it gets 6 times more throughput in the case of the constant probabilities strategy.

The fairness behavior of the proportional drop strategy has already appeared in [10], where the flow with 3 times smaller RTT gets 2.75 times more throughput. Comparing to these results we see that, in terms of fairness, the LTL strategy gives the best results whereas the worse performance is provided by the fixed loss probabilities strategy.

The behavior of the throughput as the ratio of $R T T$ goes to zero is in particular interesting. The throughput of the long flow and its share of the throughput tend to zero in the constant loss strategy, as well as with the proportional strategy [9, Sec. 7-8], whereas it tends to a positive constant under the LTL strategy.

Note that the fact that we obtain different average throughput sharing under different policies reflects the fact that, in contrast to the symmetric case, the throughput is not invariant with respect to the strategy in the general asymmetric case.

\section{The symmetric case: invariance of the throughput for a general strategy}

Consider now a general strategy for deciding which flow will decrease its rate when capacity is reached. The decrease is by a constant $\beta$ and the increase rate is $\eta$. We still restrict ourselves to the symmetric case of two flows, and assume that one and only one flow decreases its rate when the capacity is reached. At such a moment, flow 1 that transmits at a rate of $y$ will decrease its rate with probability $f(y)$ and flow 2 will decrease its rate with probability 
$1-f(y)$. We assume that the rate process of both flows is in a stationary ergodic regime. In particular we shall focus again on $Y_{n}^{(1)}$, the rate of flow 1 just before a rate decrease occurs.

\subsection{The Markov chain}

We focus on flow $1 ; Y_{n}:=Y_{n}^{(1)}$ is a semi-Markov process. If the state at time $T_{n}$ is $Y_{n}=y$, then

- If flow 1 is the one to decrease its rate (this occurs with probability $f(y)$ ) then we shall have at $T_{n+1}$

$$
Y_{n+1}^{(1)}=\beta y+\eta \tau_{n+1}, \quad Y_{n+1}^{(2)}=C-y+\eta \tau_{n+1}
$$

Since the sum of the rates of the flows at that time is $C$, we obtain $\tau_{n+1}=$ $y(1-\beta) /(2 \eta)$, and thus

$$
Y_{n+1}=y\left(\frac{1+\beta}{2}\right) .
$$

Finally, the surface $S:=S^{(1)}$ is given by

$$
S=\frac{1}{2}\left(\beta Y_{n}+Y_{n+1}\right) \tau_{n+1}=y^{2} \frac{(1+3 \beta)(1-\beta)}{8 \eta}
$$

- If flow 2 is the one to decrease its rate (this occurs with probability $1-f(y)$ ) then

$$
Y_{n+1}^{(1)}=y+\eta \tau_{n+1}, \quad Y_{n+1}^{(2)}=\beta(C-y)+\eta \tau_{n+1} .
$$

Since the sum of the rates of the flows at that time is $C$, we obtain $\tau_{n+1}=$ $(C-y)(1-\beta) /(2 \eta)$, and thus

$$
Y_{n+1}=y \frac{1+\beta}{2}+C \frac{1-\beta}{2}
$$

Next we compute $S$ :

$$
S=\frac{1}{2}\left(Y_{n}+Y_{n+1}\right) \tau_{n+1}=\frac{1-\beta}{8 \eta}\left(-(3+\beta) y^{2}+2 C(1+\beta) y+C^{2}(1-\beta)\right) .
$$

Below we shall use $Y$ to denote a random variable distributed like $Y_{n}$ at steady state. Similarly we shall use the notation $\tau$ to denote $\tau_{n+1}$ at steady state. 


\subsection{Expectations}

By symmetry we have $\mathbb{E}[Y]=C / 2$, and also clearly $\mathbb{E}[f(Y)]=1 / 2$. Using the previous expressions for $\tau$ and then taking expectation we obtain at steady state

$$
\begin{aligned}
\mathbb{E}[\tau] & =\mathbb{E}\left[Y \frac{1-\beta}{2 \eta} f(Y)+\frac{(C-Y)(1-\beta)}{2 \eta}(1-f(Y))\right] \\
& =(1-\beta) \mathbb{E}[Y f(Y)] / \eta
\end{aligned}
$$

This can also be obtained alternatively by an up-down crossing argument: If we denote by $Z_{n}$ the rate of flow 1 just after $T_{n}$, then the expected decrease in rate at $T_{n}$ is

$$
\mathbb{E}\left[Y_{n}-Z_{n}\right]=(1-\beta) \mathbb{E}[Y f(Y)]
$$

The average increase in the rate $\eta \mathbb{E}[\tau]$ should compensate for the average decrease in the rate, from which we obtain (12). Next we express the expectation of the surface:

$$
\begin{aligned}
\mathbb{E}[S]= & \frac{1-\beta}{8 \eta} \mathbb{E}\left[(1+3 \beta) Y^{2} f(Y)+\left(-(3+\beta) Y^{2}\right.\right. \\
& \left.\left.+2 C(1+\beta) Y+C^{2}(1-\beta)\right)(1-f(Y))\right] \\
= & \frac{1-\beta}{8 \eta}\left(-(3+\beta) \mathbb{E}\left[Y^{2}\right]+4(1+\beta) \mathbb{E}\left[Y^{2} f(Y)\right]\right. \\
& \left.-2 C(1+\beta) \mathbb{E}[Y f(Y)]+C^{2} \frac{3+\beta}{2}\right) .
\end{aligned}
$$

Proposition 2 The average throughput of a flow in a symmetric network of two flows is given by

$$
\bar{Y}=\frac{1+\beta}{3+\beta} C,
$$

independent of the sampling function $f$.

Proof: We need to compute $\mathbb{E}[S] / \mathbb{E}[\tau]$, but for that we first need to compute several unknowns: $\mathbb{E}[Y f(Y)], \mathbb{E}\left[Y^{2}\right]$ and $\mathbb{E}\left[Y^{2} f(Y)\right]$. In order to obtain them, we shall also need to obtain the unknown $\mathbb{E}\left[Y^{3}\right]$.

Our first relation between the unknowns is obtained by writing that $\mathbb{E}\left[\left(Y_{n}\right)^{2}\right]=$ $\mathbb{E}\left[\left(Y_{n+1}\right)^{2}\right]$ and using our previous expressions to write $Y_{n+1}$ in terms of $Y_{n}$ :

$$
\mathbb{E}\left[Y^{2}\right]=\mathbb{E}\left[\left(\frac{1+\beta}{2}\right)^{2} Y^{2} f(Y)+\frac{1}{4}((1+\beta) Y+(1-\beta) C)^{2}(1-f(Y))\right]
$$

Note that the term $\mathbb{E}\left[Y^{2} f(Y)\right]$ cancels out here, and we obtain a simple relation between the unknowns $\mathbb{E}\left[Y^{2}\right]$ and $\mathbb{E}[Y f(Y)]$. 


$$
\mathbb{E}\left[Y^{2}\right]\left(1-\left(\frac{1+\beta}{2}\right)^{2}\right)=\frac{C^{2}}{8}(1-\beta)(3+\beta)-\frac{C}{2}\left(1-\beta^{2}\right) \mathbb{E}[Y f(Y)]
$$

To obtain an expression for $\mathbb{E}\left[Y^{2} f(Y)\right]$ we use the fact that at steady state $\mathbb{E}\left[\left(Y_{n}\right)^{3}\right]=\mathbb{E}\left[\left(Y_{n+1}\right)^{3}\right]$ and substitute our previous expressions to write $Y_{n+1}$ in terms of $Y_{n}$ :

$$
\mathbb{E}\left[Y^{3}\right]=\mathbb{E}\left[\left(\frac{1+\beta}{2}\right)^{3} Y^{3} f(Y)+\frac{1}{8}((1+\beta) Y+(1-\beta) C)^{3}(1-f(Y))\right] .
$$

Note that the term $\mathbb{E}\left[Y^{3} f(Y)\right]$ cancels out here, and we obtain a relation between all the unknowns $\mathbb{E}\left[Y^{3}\right], \mathbb{E}\left[Y^{2}\right], \mathbb{E}\left[Y^{2} f(Y)\right]$ and $\mathbb{E}[Y f(Y)]$ :

$$
\begin{aligned}
\mathbb{E}\left[Y^{3}\right]\left(1-\frac{1}{8}(1+\beta)^{3}\right)= & \frac{3 C}{8}(1+\beta)^{2}(1-\beta) \mathbb{E}\left[Y^{2}\right] \\
& -\frac{3 C}{8}(1+\beta)^{2}(1-\beta) \mathbb{E}\left[Y^{2} f(Y)\right] \\
& -\frac{3 C^{2}}{8}(1+\beta)(1-\beta)^{2} \mathbb{E}[Y f(Y)] \\
& +\frac{C^{3}}{8}(1-\beta)^{2}(2+\beta)
\end{aligned}
$$

Another relation is obtained by using the fact that by symmetry $\mathbb{E}\left[Y^{3}\right]=$ $\mathbb{E}\left[(C-Y)^{3}\right]$ (since $C-Y$ is the rate of the second flow). This gives

$$
\mathbb{E}\left[Y^{3}\right]=\frac{1}{2}\left(3 C \mathbb{E}\left[Y^{2}\right]-3 \frac{C^{3}}{2}+C^{3}\right)=\frac{1}{2}\left(3 C \mathbb{E}\left[Y^{2}\right]-\frac{C^{3}}{2}\right) .
$$

We thus have three equations with four unknowns. If we had four independent equations this would have given us a single solution for the unknowns. But noting that the coefficients of these unknowns do not depend on the function $f$, this would mean that the unknowns do not depend on $f$. This is however not the case, since we already saw above that $\mathbb{E}\left[Y^{2}\right]$ is different for different functions $f$. We shall carry however the calculations in order to check whether the average rates will turn out not to depend on $f$.

Using the previous relations, we can express $\mathbb{E}[S]$ in terms of $\mathbb{E}[Y f(Y)]$ by

$$
\mathbb{E}[S]=\frac{(1-\beta)(1+\beta) \mathbb{E}[Y(f(Y)] C}{\eta(3+\beta)} .
$$

Using the expression (12) of $\mathbb{E}[\tau]$, we obtain the result. 


\section{The symmetric case: second moment of the throughput}

Even if all possible loss strategies provide the same average throughput in steady-state in the symmetric case we can wonder about the variability of the throughput. In real-time applications that may use AIMD protocols in order to be TCP-friendly, it is clearly advantageous to have the lowest possible throughput variability.

The following Proposition gives a general expression for the second moment of the throughput. As will be seen, this expression is not invariant any more, in contrast to the first moment.

Proposition 3 Let $\mathbb{E}\left[S_{2}\right]$ denote the mean cumulative of the square throughput between two loss epochs. The (average) second moment of throughput is

$$
\frac{\mathbb{E}\left[S_{2}\right]}{\mathbb{E}[\tau]}=\frac{1}{3}\left(1+\beta+\beta^{2}\right) \frac{\mathbb{E}\left[Y^{3} f(Y)\right]}{\mathbb{E}[Y f(Y)]} .
$$

Proof: The mean cumulative of the square throughput between two loss epochs is given by

$$
S_{2}=\frac{1}{3} \frac{\left(Y_{n+1}\right)^{3}-\left(\beta Y_{n}\right)^{3}}{Y_{n+1}-\beta Y_{n}} \tau_{n+1}=\frac{1}{3}\left(\beta^{2} Y_{n}^{2}+\beta Y_{n} Y_{n+1}+Y_{n+1}^{2}\right) \tau_{n+1}
$$

if the loss is experienced at time $n$ by session 1 , and

$$
S_{2}=\frac{1}{3} \frac{\left(Y_{n+1}\right)^{3}-\left(Y_{n}\right)^{3}}{Y_{n+1}-Y_{n}} \tau_{n+1}=\frac{1}{3}\left(Y_{n}^{2}+Y_{n} Y_{n+1}+Y_{n+1}^{2}\right) \tau_{n+1}
$$

otherwise. Using the previous results we have

$$
\begin{aligned}
\mathbb{E}\left[S_{2}\right]= & \frac{1-\beta}{6 \eta} \mathbb{E}\left[\left(\beta^{2} Y^{2}+\beta\left(\frac{1+\beta}{2}\right) Y^{2}+Y^{2}\left(\frac{1+\beta}{2}\right)^{2}\right) Y f(Y)\right. \\
& +\left(Y^{2}+\frac{Y}{2}(Y(1+\beta)+C(1-\beta))+\frac{1}{4}(Y(1+\beta)+C(1-\beta))^{2}\right) \\
& \times(C-Y)(1-f(Y))] \\
= & \frac{1-\beta}{24 \eta}\left(\left(8+8 \beta+8 \beta^{2}\right) \mathbb{E}\left[Y^{3} f(Y)\right]-\left(7+4 \beta+\beta^{2}\right) \mathbb{E}\left[Y^{3}\right]\right. \\
& +C\left(3+6 \beta+3 \beta^{2}\right) \mathbb{E}\left[Y^{2}\right]-C\left(3+6 \beta+3 \beta^{2}\right) \mathbb{E}\left[Y^{2} f(Y)\right] \\
& \left.-C^{2}\left(3-3 \beta^{2}\right) \mathbb{E}[Y f(Y)]+C^{3}\left(2-\beta-\beta^{2}\right)\right) .
\end{aligned}
$$

Following the proof of Proposition 2, the only unknowns left are $\mathbb{E}[Y f(Y)]$ 
and $\mathbb{E}\left[Y^{3} f(Y)\right]$ and we get

$$
\mathbb{E}\left[S_{2}\right]=\frac{1}{3 \eta}(1-\beta)\left(1+\beta+\beta^{2}\right) \mathbb{E}\left[Y^{3} f(Y)\right] .
$$

We then obtain the result.

Since we still have two unknowns, one could argue that their ratio is constant. Actually, it is not the case from the following proposition where we compare the second order moment for the three loss strategies (constant, proportional or largest flow).

Proposition 4 Let $\beta=1 / 2$. Using the constant loss probability scheme, we get

$$
Q_{c s t}=\frac{\mathbb{E}\left[S_{2}\right]}{\mathbb{E}[\tau]}=\frac{5}{24} C^{2} \approx 0.2083 C^{2}
$$

whereas when the loss is applied to the largest flow (LTL strategy), we have

$$
Q_{l t l}=\frac{\mathbb{E}\left[S_{2}\right]}{\mathbb{E}[\tau]}=\frac{4}{21} C^{2} \approx 0.1905 C^{2}
$$

and the scheme with proportional losses gives

$$
Q_{\text {pro }}=\frac{\mathbb{E}\left[S_{2}\right]}{\mathbb{E}[\tau]}=\frac{679}{3396} C^{2} \approx 0.19994 C^{2} .
$$

We see from the proposition that in the symmetric case, the LTL strategy is to be preferred (in terms of lower second moment), whereas the strategy of fixed loss probability has the worst performance.

Proof of Proposition 4: Consider the scheme with constant loss probability. Since $f(Y)=1 / 2$, we have $\mathbb{E}\left[Y^{j} f(Y)\right]=\mathbb{E}\left[Y^{j}\right] / 2 \forall j$. From Equation (13), we get $\mathbb{E}\left[Y^{2}\right]=2 C^{2} / 7$. Then, from (14), $\mathbb{E}\left[Y^{3}\right]=5 C^{3} / 28$, leading to the result.

If we look at the scheme where the loss is experienced to the largest flow in its periodic behaviour, we can directly get $\mathbb{E}\left[S_{2}\right]$ from the mean cumulative of the square throughput between loss epochs $T_{n}$ and $T_{n+2}$ by

$$
2 \mathbb{E}\left[S_{2}\right]=\int_{0}^{2 C / 7}(x+2 C / 7)^{2} d x=\frac{8}{147} C^{3} .
$$

Since $\mathbb{E}[\tau]=C / 7$, we get the result.

Let us now look at the scheme with proportional losses. We have $F(Y)=Y / C$. From $[9]$, we have $\mathbb{E}[Y]=C / 2, \mathbb{E}\left[Y^{2}\right]=7 C^{2} / 26, \mathbb{E}\left[Y^{3}\right]=2 C^{3} / 13, \mathbb{E}\left[Y^{4}\right]=$ 
$679 C^{4} / 7358$. This leads to $E[Y f(Y)]=7 C / 26$ and $E\left[Y^{3} f(Y)\right]=679 C^{3} / 7358$, which gives the result.

\section{Discussion and future research}

We have presented in this paper various loss strategies that determine which flow will lose a packet when a congestion occurs. We have shown that such loss strategies may have a considerable impact on the throughput variability (which may be an important performance measure in real-time applications that use AIMD protocols to be TCP-friendly) but that they all lead to the same average throughput in the special case of a symmetric network with two flows. Among three specific strategies that we introduced, we have shown in the above setting that the LTL strategy (i.e., the strategy that drops a packet from the flow with highest throughput) has the best performance in terms of throughput variability, and moreover, it guarantees a positive share of the throughput even when the RTT of one of the flows becomes arbitrarily large.

The mathematical study of the sharing of bandwidth under various loss strategies turns out to be quite involved. So far we have not been able to get explicit expressions for the asymmetric network with two flows when the LTL or the proportional loss strategies are used. We have provided however an (involved) explicit expression for the throughput for the case of constant loss strategy. For the symmetric case, however, we have obtained an explicit expression for the throughput under an arbitrary loss strategy.

The cases of more than one link and more than two flows, however interesting, are beyond the scope of this paper. In particular, we indeed use the model of [7] where there is a single bottleneck, common to all the flows that traverse it. So far we have not been able to apply our analytical approach to study more than two flows; this is left for future research.

Many open problems remain: 1 . Is there any probabilistic argument that can explain the invariance of the average throughput in the loss strategy phenomenon in the case of two flows? 2. Does the invariance of the throughput holds for the case of more than two competing symmetric flows? 3. What is the reason for the fractal behavior of the throughput sharing under LTL? 4. How to implement LTL? Note that a desirable way of implementation should be stateless, and it should make use only of local information available at the bottleneck element. 


\section{References}

[1] E. Altman, R. El Azouzi, D. Ros, B. Tuffin, Loss Strategies for Competing TCP/IP Connections, in: Proceedings of NETWORKING 2004, no. 3042 in Lecture Notes in Computer Science, Springer, Athens, Greece, 2004, pp. 926937.

[2] J. Padhye, V. Firoiu, J. Kurose, D. Towsley, Modeling TCP Throughput: A Simple Model and its Empirical Validation, in: Proceedings of ACM SIGCOMM, 1998, pp. 303-314.

[3] P. Brown, Resource Sharing of TCP Connections with Different Round Trip Times, in: Proceedings of IEEE Infocom, 2000.

[4] T. Lakshman, U. Madhow, The Performance of TCP/IP for Networks with High Bandwidth-Delay Products and Random Loss, IEEE/ACM Transactions on Networking 5 (3) (1997) 336-350.

[5] O. Ait-Hellal, E. Altman, D. Elouadghiri, M. Erramdani, N. Mikou, Performance of TCP/IP: The Case of Two Controlled Sources, in: Proceedings of ICCC'97, Cannes, 1997, pp. 469-477.

[6] L. Zhang, S. Shenker, D. Clark, Observations on the Dynamics of a Congestion Control Algorithm: The Effects of Two-Way Traffic, in: Proceedings of ACM SIGCOMM, 1991.

[7] F. Baccelli, D. Hong, AIMD, Fairness and Fractal Scaling of TCP Traffic, in: Proceedings of IEEE INFOCOM, 2002.

[8] M. Vojnovic, J. Y. Le-Boudec, Some Observations on Equation-Based Rate Control, in: Proceedings of ITC-17, Salvador da Bahia, 2001.

[9] E. Altman, T. Jiménez, R. Núñez-Queija, Analysis of Two Competing TCP/IP Connections, Performance Evaluation 49 (1) (2002) 43-56.

[10] E. Altman, C. Barakat, E. Laborde, P. Brown, D. Collange, Fairness Analysis of TCP/IP, in: Proceedings of IEEE CDC'00, Sydney, 2000.

[11] L. DaSilva, Pricing of QoS-Enabled Networks: A Survey, IEEE Communications Surveys \& Tutorials 3 (2).

[12] M. Falkner, M. Devetsikiotis, I. Lambadaris, An Overview of Pricing Concepts for Broadband IP Networks, IEEE Communications Surveys \& Tutorials 3 (2).

[13] B. Tuffin, Charging the Internet without Bandwidth Reservation: An Overview and Bibliography of Mathematical Approaches, Journal of Information Science and Engineering 19 (5) (2003) 765-786.

[14] E. Altman, K. Avratchenkov, C. Barakat, A Stochastic Model of TCP/IP with Stationary Random Losses, in: Proceedings of ACM SIGCOMM, 2000.

[15] F. Baccelli, D. Hong, Interaction of TCP Flows as Billiards, Research Report RR-4437, INRIA (Apr. 2002). 


\section{A Proof of Proposition 1}

Using (3), the recursive equation (1) becomes

$$
Y_{n+1}^{(i)}=\gamma_{n}^{(i)} Y_{n}^{(i)}\left(1-\frac{\eta_{i}}{\sum_{j=1}^{N} \eta_{j}}\right)-\eta_{i} \frac{\sum_{j \neq i} \gamma_{n}^{(j)} Y_{n}^{(j)}}{\sum_{j=1}^{N} \eta_{j}}+C \frac{\eta_{i}}{\sum_{j=1}^{N} \eta_{j}} .
$$

Since $N=2$, we have

$$
Y_{n}^{(2)}=C-Y_{n}^{(1)}
$$

and $\forall i \in\{1,2\}$ and $j \in\{1,2\}$ with $j \neq i$,

$$
Y_{n+1}^{(i)}=\gamma_{n}^{(i)} Y_{n}^{(i)}\left(1-\frac{\eta_{i}}{\sum_{k=1}^{2} \eta_{k}}\right)-\eta_{i} \frac{\gamma_{n}^{(j)}\left(C-Y_{n}^{(i)}\right)}{\sum_{k=1}^{2} \eta_{k}}+C \frac{\eta_{i}}{\sum_{k=1}^{2} \eta_{k}}
$$

leading to

$$
Y_{n+1}^{(i)}=Y_{n}^{(i)}\left(\gamma_{n}^{(i)}-\left(\gamma_{n}^{(i)}-\gamma_{n}^{(j)}\right) \frac{\eta_{i}}{\sum_{k=1}^{2} \eta_{k}}\right)+C \frac{\eta_{i}}{\sum_{k=1}^{2} \eta_{k}}\left(1-\gamma_{n}^{(j)}\right)
$$

If we define

and

$$
B_{n}^{(i)}=\gamma_{n}^{(i)}-\left(\gamma_{n}^{(i)}-\gamma_{n}^{(j)}\right) \frac{\eta_{i}}{\sum_{k=1}^{2} \eta_{k}}
$$

Equation (A.3) can be written

$$
A_{n}^{(i)}=C \frac{\eta_{i}}{\sum_{k=1}^{2} \eta_{k}}\left(1-\gamma_{n}^{(j)}\right),
$$

$$
Y_{n+1}^{(i)}=Y_{n}^{(i)} B_{n}^{(i)}+A_{n}^{(i)} .
$$

In steady-state, we get

$$
\mathbb{E}\left(Y^{(i)}\right)=\frac{\mathbb{E}\left(A^{(i)}\right)}{1-\mathbb{E}\left(B^{(i)}\right)} .
$$

To compute the mean throughput of session $i$, we have

$$
\bar{Y}^{(i)}=\frac{\mathbb{E}\left(S^{(i)}\right)}{\mathbb{E}(\tau)},
$$

where $\mathbb{E}\left[S^{(i)}\right]$ is the average cumulated throughput between two congestion epochs.

First, since $\left(\left(a_{n}^{(1)}, a_{n}^{(2)}\right)\right)_{n}$ is a sequence of independent random vectors, $\gamma_{n}^{(i)}$ and $Y_{n}^{(i)}$ are independent and

$$
\mathbb{E}[\tau]=\frac{C-\mathbb{E}\left[\gamma^{(1)}\right] \mathbb{E}\left[Y^{(1)}\right]-\mathbb{E}\left[\gamma^{(2)}\right] \mathbb{E}\left[Y^{(2)}\right]}{\sum_{k=1}^{2} \eta_{k}} .
$$


Let

Using

$$
\xi_{i}=\frac{\eta_{i}}{\sum_{k=1}^{2} \eta_{k}}
$$

$$
\begin{aligned}
\mathbb{E}\left[\gamma^{(i)}\right] & =1-p_{i}+\beta_{i} p_{i} \\
\mathbb{E}\left[A^{(i)}\right] & =C \xi_{i}\left(1-\mathbb{E}\left[\gamma^{(j)}\right]\right)=C \xi_{i} p_{j}\left(1-\beta_{j}\right) \\
\mathbb{E}\left[B^{(i)}\right] & =\left(1-\xi_{i}\right) \mathbb{E}\left[\gamma^{(i)}\right]+\xi_{i} \mathbb{E}\left[\gamma^{(j)}\right]=\xi_{j} \mathbb{E}\left[\gamma^{(i)}\right]+\xi_{i} \mathbb{E}\left[\gamma^{(j)}\right] \\
& =1-\frac{\left(1-\beta_{i}\right) \eta_{j} p_{i}+\left(1-\beta_{j}\right) \eta_{i} p_{j}}{\sum_{k=1}^{2} \eta_{k}} \\
\mathbb{E}\left[Y^{(i)}\right] & =\frac{\mathbb{E}\left[A^{(i)}\right]}{1-\mathbb{E}\left[B^{(i)}\right]}=\frac{C \eta_{i} p_{j}\left(1-\beta_{j}\right)}{\left(1-\beta_{2}\right) \eta_{1} p_{2}+\left(1-\beta_{1}\right) \eta_{2} p_{1}}
\end{aligned}
$$

Note that $\mathbb{E}\left[B^{(i)}\right]=\mathbb{E}\left[B^{(j)}\right]$ do not depend on the session and can then be written $\mathbb{E}[B]$.

We can deduce that

$$
\mathbb{E}[\tau]=C \frac{\left(1-\beta_{1}\right)\left(1-\beta_{2}\right) p_{1} p_{2}}{\left(1-\beta_{2}\right) \eta_{1} p_{2}+\left(1-\beta_{1}\right) \eta_{2} p_{1}} .
$$

We now need to compute $\mathbb{E}\left[S^{(i)}\right]$ in terms of $p_{1}$ and $p_{2}$. If we note $S_{n}^{(i)}$, the cumulated throughput between $n$-th and $n+1$-th congestion epochs, we have

$$
\begin{aligned}
S_{n}^{(i)}= & \frac{1}{2}\left(Y_{n+1}^{(i)}+\gamma_{n}^{(i)} Y_{n}^{(i)}\right) \tau_{n+1} \\
= & \frac{1}{2}\left(Y_{n}^{(i)} B_{n}^{(i)}+A_{n}^{(i)}+\gamma_{n}^{(i)} Y_{n}^{(i)}\right) \frac{C-\gamma_{n}^{(i)} Y_{n}^{(i)}-\gamma_{n}^{(j)} Y_{n}^{(j)}}{\sum_{k=1}^{2} \eta_{k}} \\
= & \frac{1}{2}\left(Y_{n}^{(i)} B_{n}^{(i)}+A_{n}^{(i)}+\gamma_{n}^{(i)} Y_{n}^{(i)}\right) \frac{\left(1-\gamma_{n}^{(j)}\right) C+\left(\gamma_{n}^{(j)}-\gamma_{n}^{(i)}\right) Y_{n}^{(i)}}{\sum_{k=1}^{2} \eta_{k}} \\
= & \left(Y_{n}^{(i)}\left(C\left(1-\gamma_{n}^{(j)}\right)\left(B_{n}^{(i)}+\gamma_{n}^{(i)}\right)+\left(\gamma_{n}^{(j)}-\gamma_{n}^{(i)}\right) A_{n}^{(i)}\right)+\left(C A_{n}^{(i)}\left(1-\gamma_{n}^{(j)}\right)\right)\right. \\
& \left.+\left(Y_{n}^{(i)}\right)^{2}\left(\left(\gamma_{n}^{(j)}-\gamma_{n}^{(i)}\right)\left(B_{n}^{(i)}+\gamma_{n}^{(i)}\right)\right)\right)\left(2 \sum_{k=1}^{2} \eta_{k}\right)^{-1}
\end{aligned}
$$

From (A.4), since $B_{n}^{(i)}$ and $Y_{n}^{(i)}$ are independent, we get

$$
\mathbb{E}\left[\left(Y_{n+1}^{(i)}\right)^{2}\right]=\mathbb{E}\left[\left(B_{n}^{(i)}\right)^{2}\right] \mathbb{E}\left[\left(Y_{n}^{(i)}\right)^{2}\right]+\mathbb{E}\left[\left(A_{n}^{(i)}\right)^{2}\right]+2 \mathbb{E}\left[Y_{n}^{(i)}\right] \mathbb{E}\left[B_{n}^{(i)} A_{n}^{(i)}\right]
$$

leading to

$$
\mathbb{E}\left[\left(Y^{(i)}\right)^{2}\right]=\frac{\mathbb{E}\left[\left(A^{(i)}\right)^{2}\right]+2 \mathbb{E}\left[Y^{(i)}\right] \mathbb{E}\left[B^{(i)} A^{(i)}\right]}{1-\mathbb{E}\left[\left(B^{(i)}\right)^{2}\right]}
$$


Using the fact that $\forall k \in\{1,2\},\left(a_{n}^{(k)}\right)^{2}=a_{n}^{(k)}$, we have

$$
\begin{aligned}
\mathbb{E}\left[\left(\gamma^{(i)}\right)^{2}\right]= & 1-2\left(1-\beta_{i}\right) p_{i}+\left(1-\beta_{i}\right)^{2} \mathbb{E}\left[\left(a^{(i)}\right)^{2}\right] \\
= & 1-\left(1-\beta_{i}^{2}\right) p_{i} \\
\mathbb{E}\left[\gamma^{(1)} \gamma^{(2)}\right]= & 1-\sum_{k=1}^{2}\left(1-\beta_{k}\right) p_{k}+\left(\prod_{k=1}^{2}\left(1-\beta_{k}\right)\right) \mathbb{E}\left[a^{(1)} a^{(2)}\right] \\
\mathbb{E}\left[\left(A^{(i)}\right)^{2}\right]= & C^{2} \xi_{i}^{2}\left(1-2 \mathbb{E}\left[\gamma^{(j)}\right]+\mathbb{E}\left[\left(\gamma^{(j)}\right)^{2}\right]\right) \\
= & C^{2} \xi_{i}^{2}\left(1-\beta_{j}\right)^{2} p_{j} \\
\mathbb{E}\left[\left(B^{(i)}\right)^{2}\right]= & \xi_{1}^{2} \mathbb{E}\left[\left(\gamma^{(2)}\right)^{2}\right]+\xi_{2}^{2} \mathbb{E}\left[\left(\gamma^{(1)}\right)^{2}\right]+2 \xi_{1} \xi_{2} \mathbb{E}\left[\gamma^{(1)} \gamma^{(2)}\right] \\
= & 1-2 \xi_{1}\left(1-\beta_{2}\right) p_{2}-2 \xi_{2}\left(1-\beta_{1}\right) p_{1} \\
& +\mathbb{E}\left[\left(\xi_{1}\left(1-\beta_{1}\right) a^{(1)}+\xi_{2}\left(1-\beta_{2}\right) a^{(2)}\right)^{2}\right] \\
\mathbb{E}\left[B^{(i)} A^{(i)}\right]= & C \xi_{i} \mathbb{E}\left[\xi_{i} \gamma^{(j)}-\xi_{i}\left(\gamma^{(j)}\right)^{2}+\xi_{j} \gamma^{(i)}-\xi_{j} \gamma^{(i)} \gamma^{(j)}\right] \\
= & C \xi_{i}\left[\left(1-\beta_{j}\right) p_{j}-\xi_{i}\left(1-\beta_{j}\right)^{2} p_{j}-\xi_{j}\left(\prod_{k=1}^{2}\left(1-\beta_{k}\right)\right) \mathbb{E}\left[a^{(1)} a^{(2)}\right]\right] \\
\mathbb{E}\left[\left(Y^{(i)}\right)^{2}\right]= & \left(C^{2} \xi_{i}^{2}\left(1-\beta_{j}\right)^{2} p_{j}\right. \\
C^{2} \xi_{i}^{2} p_{j}(1- & \left.\beta_{j}\right)\left[\left(1-\beta_{j}\right) p_{j}-\xi_{i}\left(1-\beta_{j}\right)^{2} p_{j}-\xi_{j}\left(\prod_{k=1}^{2}\left(1-\beta_{k}\right)\right) \mathbb{E}\left[a^{(1)} a^{(2)}\right]\right] \\
+\xi_{1} p_{2}+\xi_{2} p_{1}-\beta_{2} \xi_{1} p_{2}-\beta_{1} \xi_{2} p_{1} & \\
\times\left(2 \xi_{1}(1-\right. & \left.\left.\beta_{2}\right) p_{2}+2 \xi_{2}\left(1-\beta_{1}\right) p_{1}-\mathbb{E}\left[\left(\xi_{1}\left(1-\beta_{1}\right) a^{(1)}+\xi_{2}\left(1-\beta_{2}\right) a^{(2)}\right)^{2}\right]\right)^{-1} \\
(\mathrm{~A} 9) &
\end{aligned}
$$

We also have

$$
\begin{aligned}
\mathbb{E}\left[\left(1-\gamma_{n}^{(j)}\right) A_{n}^{(i)}\right] & =C \xi_{i} \mathbb{E}\left[\left(1-\gamma^{(j)}\right)^{2}\right] \\
& =C \xi_{i}\left(1-\beta_{j}\right)^{2} p_{j}
\end{aligned}
$$

$$
\begin{aligned}
\mathbb{E}\left[\left(\gamma_{n}^{(j)}-\right.\right. & \left.\left.\gamma_{n}^{(i)}\right)\left(B_{n}^{(i)}+\gamma_{n}^{(i)}\right)\right]= \\
= & \mathbb{E}\left[\left(\left(1-\beta_{i}\right) a^{(i)}-\left(1-\beta_{j}\right) a^{(j)}\right)\right. \\
& \left.\times\left(1-\xi_{j}\left(1-\beta_{i}\right) a^{(i)}-\xi_{i}\left(1-\beta_{j}\right) a^{(j)}+1-\left(1-\beta_{i}\right) a^{(i)}\right)\right] \\
= & \xi_{i}\left(1-\beta_{j}\right)^{2} p_{j}-\left(1+\xi_{j}\right)\left(1-\beta_{i}\right)^{2} p_{i}-2\left(1-\beta_{j}\right) p_{j} \\
& +2 \xi_{j}\left(1-\beta_{j}\right)\left(1-\beta_{i}\right) \mathbb{E}\left[a^{(i)} a^{(j)}\right]+2\left(1-\beta_{i}\right) p_{i}
\end{aligned}
$$




$$
\begin{aligned}
\mathbb{E}[C & \left.\left(1-\gamma_{n}^{(j)}\right)\left(B_{n}^{(i)}+\gamma_{n}^{(i)}\right)+\left(\gamma_{n}^{(j)}-\gamma_{n}^{(i)}\right) A_{n}^{(i)}\right]= \\
= & \mathbb{E}\left[C\left(1-\beta_{j}\right) a^{(j)}\left[2-\left(1+\xi_{j}\right)\left(1-\beta_{i}\right) a^{(i)}-\xi_{i}\left(1-\beta_{j}\right) a^{(j)}\right]\right. \\
& \left.\quad+\left(\left(1-\beta_{i}\right) a^{(i)}-\left(1-\beta_{j}\right) a^{(j)}\right) C \xi_{i}\left(1-\beta_{j}\right) a^{(j)}\right] \\
= & C\left(2\left(1-\beta_{j}\right) p_{j}-2 \xi_{i}\left(1-\beta_{j}\right)^{2} p_{j}-2 \xi_{j}\left(1-\beta_{j}\right)\left(1-\beta_{i}\right) \mathbb{E}\left[a^{(i)} a^{(j)}\right]\right) .
\end{aligned}
$$

Inserting (A.9), (A.10),(A.11), and (A.12) into (A.7), we get an expression of $\mathbb{E}\left[S^{(i)}\right]$. Dividing by the expression (A.6) of $\mathbb{E}[\tau]$, and denoting $p_{12}=$ $\mathbb{E}\left(a^{(1)} a^{(2)}\right), \alpha_{i}=1-\beta_{i}, p_{i}^{\prime}=\alpha_{i} p_{i} \forall i=1,2$ and $p_{12}^{\prime}=\alpha_{1} \alpha_{2} p_{12}$, we obtain the result. 